\title{
Onset of Linear Instability in a Complex Plasma with Cairns Distributed Electrons
}

\author{
I. Habumugisha ${ }^{1,2^{*}}$, S. K. Anguma ${ }^{3}$, E. Jurua ${ }^{1}$, N. Noreen ${ }^{4}$ \\ ${ }^{1}$ Department of Physics, Mbarara University of Science and Technology, Mbarara, Uganda \\ ${ }^{2}$ Department of Physics, Islamic University in Uganda, Mbale, Uganda \\ ${ }^{3}$ Department of Physics, Muni University, Arua, Uganda \\ ${ }^{4}$ Department of Physics, Forman Christian College (Chartered University), Lahore, Pakistan \\ Email: "hisaac08@gmail.com
}

Received 12 December 2015; accepted 4 March 2016; published 7 March 2016

Copyright (C) 2016 by authors and Scientific Research Publishing Inc.

This work is licensed under the Creative Commons Attribution International License (CC BY). http://creativecommons.org/licenses/by/4.0/

(c) (i) Open Access

\section{Abstract}

A rigorous theoretical investigation of linear dust ion acoustic (DIA) solitary waves in an unmagnetized complex plasma consisting of ion and ion beam fluids, nonthermal electrons that are Cairns distributed and immobile dust particles were undertaken. It was found out that, for large beam speeds, three stable modes propagated as solitary waves in the beam plasma. These were the "Fast", "Slow" and "Ion-acoustic" modes. For two stream instability to occur between ion and ion beam, it is shown that $(\omega / k) \rightarrow \sqrt{3 \sigma_{i}}$ or when $\left(\omega / k-u_{b 0}\right) \rightarrow \sqrt{3 \sigma_{b}}$.

\section{Keywords}

Linear Instability, Complex Plasma, Cairns Distributed Electrons

\section{Introduction}

In a complex plasma, ion beam can significantly affect the propagation charateristics of solitary waves [1]. The presence of streaming ion beams excites ion-ion instability as a result of counter streams.

In the initial study by [2], ion beam dynamics were studied with Boltzmann distributed electrons using the standard reductive pertubation technique [2]. A year later, Misra and Adhikary studied both linear and non linear propagation of large amplitude DIA waves using theoretical and numerical approaches [3]. It was found out that three stable waves, i.e., the "Fast" and "Slow" ion-beam modes and "Ion-acoustic" modes can exist. In all these studies the electrons are Boltzmann distributed. However, several other electron populations follow the Cairns

${ }^{*}$ Corresponding author.

How to cite this paper: Habumugisha, I., Anguma, S.K., Jurua, E. and Noreen, N. (2016) Onset of Linear Instability in a Complex Plasma with Cairns Distributed Electrons. International Journal of Astronomy and Astrophysics, 6, 1-7. 
distribution [4].

For a population with excess fast particles, the Cairns distribution was introduced by Cairns et al. (1995) to analyse the effect of particles on solitary waves [5]. Since then Cairns distribution is often utilized in theoretical studies as it exhibits an enhanced high energy tail, superimposed on a Maxwellian-like low energy component (as often observed in space). It was shown that with a non-thermal electron population, the nature of ion sound solitary structures may change, and solitons with both positive and negative density pertubations can exist [4]. It therefore serves as a useful theoretical model for the family of such non-Maxiwellian or non-thermal space plasmas and it has been used by quite a number of authors, e.g., [6] [7].

The Cairns distribution is often given as [4],

$$
F_{e}(v)=\frac{N_{e 0}}{\left(2 \pi V_{t e}^{2}\right)^{1 / 2}} \frac{\left[1+\alpha\left(v / V_{t e}\right)^{4}\right]}{(1+3 \alpha)} \exp \left[\frac{-\left(v / V_{t e}\right)^{2}}{2}\right],
$$

where $N_{e 0}$ is the equilibrium electron number density; $v$ is the electron speed; $V_{t e}$ is the electron thermal velocity and $\alpha$ is a constant.

The Cairn's distribution function is shown in Figure 1 for different values of $\alpha$.

It can be clearly seen that, for $\alpha=0$, the distribution reduces to the Maxwellian distribution function. Also, for large values of $\alpha$, the Gaussian form is deformed and the distribution function develops "wings", thus becoming multi-peaked. This may lead to beam instability and as a result, the Cairns distribution is not a good model for coherent non-linear structures such as solitary waves and double layers for higher values of $\alpha$ [5] [6]. For convenience, introducing the parameter $\beta=4 \alpha /(1+3 \alpha)$, it is seen that by allowing $\alpha$ to vary from 0 to $\infty, \beta$ is restricted to $0 \leq \beta<4 / 3$. Thus the upper limit of $\beta$ was set at $4 / 7 \simeq 0.571$ [6].

When the values are above 0.571 , the Cairns distribution ceases to be monotonically decreasing. The critical values of $\beta$ corresponds to $\alpha=0.25$. Thus, the Cairns distribution is appropriate only for a limited range of the parameter $\alpha$, deviating from the Maxwellian distribution function. From the above, one can note that the prescence of non-thermally distributed electrons gives rise to changes in the structured nature of solitary waves [7].

\section{Description of the Model}

In this model we considered a collisionless, un-magnetized plasma model that consists of non-thermally distributed electrons that follow the Cairns distribution and negatively charged dust particles that are stationary. In addition, it consists of inertial warm ions and ion-beams of equal mass. The massive dust grains are considered stationary with no charge fluctuations and therefore, will only affect the equilibrium charge neutrality.

Charge neutrality at equilibrium requires that

$$
\sum q_{j} N_{j 0}=0, \text { i.e., } \quad N_{i 0}+N_{b 0}=N_{e 0}+Z_{d} N_{d 0},
$$

where $N_{j 0}\left(q_{j}\right)$ is the equilibrium charge density of the $j^{\text {th }}$ species [i.e., $j=i$ (for ions), $j=b$ (for ion

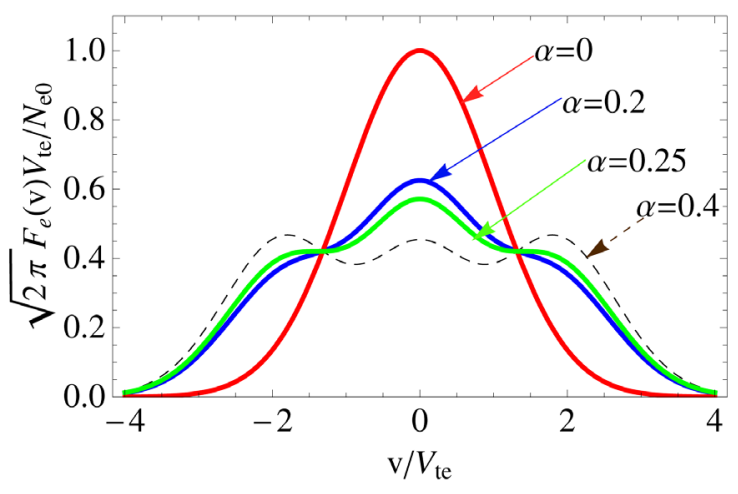

Figure 1. Plot of $\sqrt{2 \pi} F_{e}(v) V_{t e} / N_{e 0}$ against $v / V_{t e}$ for different values of $\alpha$. 
beam) and $j=e$ (for electrons)] and $N_{d 0}\left(-Z_{d} e\right)$ is the equilibrium density (charge) of the dust particles, and $Z_{d}$ is the size of the dust charge. The dynamics of warm and inertial ion and ion-beam is governed by the fluid equations, i.e., the continuity, momentum, and the adiabatic pressure equations, which in un-normalized quantities are, respectively, given by

$$
\begin{gathered}
\frac{\partial N_{j}}{\partial t^{\prime}}+\frac{\partial}{\partial x^{\prime}}\left(N_{j} V_{j}\right)=0 \\
\frac{\partial V_{j}}{\partial t^{\prime}}+V_{j} \frac{\partial V_{j}}{\partial x^{\prime}}+\frac{q_{j}}{m_{j}} \frac{\partial \phi}{\partial x^{\prime}}+\frac{1}{N_{j} m_{j}} \frac{\partial P_{j}}{\partial x^{\prime}}=0, \\
\frac{\partial P_{j}}{\partial t^{\prime}}+V_{j} \frac{\partial P_{j}}{\partial x^{\prime}}+\gamma P_{j} \frac{\partial V_{j}}{\partial x^{\prime}}=0
\end{gathered}
$$

here, $\gamma=3$ is the ratio of specific heat capacities for an adiabatic fluid; $N_{j}, V_{j}$ and $P_{j}$ are the un-normalized number density, ion/ion beam fluid speeds, and thermal pressure for species, $j$, respectively; $m_{j}\left(q_{j}\right)$ is the ion/ion beam mass (charge) and $x^{\prime}\left(t^{\prime}\right)$ is the un-normalized space (time) variable. Thus the quasi-neutral assumption, in Equation (2), and the system of Equations (3), (4), and (5) will be closed by Poisson's equation expressed as

$$
\varepsilon_{0} \frac{\partial^{2} \varphi}{\partial x^{\prime 2}}=e\left(N_{e}+Z_{d} N_{d 0}-N_{i}-N_{b}\right)
$$

where $\varepsilon_{0}$ is the permittivity of free space.

\section{Derivation of the Dispersion Relation}

The basic equations i.e., Equations (3), (4), and (5) were first linearlized. During the linearization process, it was assumed that the pertubations vary as $\sim \exp (i k x-i \omega t)$. This implied that $\partial / \partial x \rightarrow i k$ and $\partial / \partial t \rightarrow-i \omega$, where $\omega$ is the angular frequency normalized by ion plasma frequency $\omega_{p i}$ and $k$ is the wave number normalized by the reciprocal of the effective Debye length $\lambda_{\text {Deff }}^{-1}$.

The electron density is obtained by integrating the Cairns distribution function as

$$
\begin{aligned}
N_{e}(\varphi) & =\int_{-\infty}^{\infty} F_{e}(v) \mathrm{d} v \\
& =N_{e 0}\left[1-\beta\left(\frac{e \varphi}{k_{B} T_{e}}\right)+\beta\left(\frac{e \varphi}{k_{B} T_{e}}\right)^{2}\right] \exp \left[\frac{e \varphi}{k_{B} T_{e}}\right],
\end{aligned}
$$

where $\varphi$ is the un-normalized electrostatic potential. Therefore, taking $\varphi \approx \varphi_{1}$ the electron density becomes,

$$
N_{e}\left(\varphi_{1}\right)=N_{e 0}\left\{1+(1-\beta)\left[\frac{e \varphi_{1}}{k_{B} T_{e}}\right]\right\} .
$$

The ion density, $N_{i}(\phi)$ or ion beam density, $N_{b}(\phi)$ are obtained by solving Equations (3), (4), and (5) simultaneously. Taking $N_{j}=N_{j 0}+N_{j 1}, V_{j}=V_{j 0}+V_{j 1}$ and $P_{j}=P_{j 0}+P_{j 1}$ with $V_{j 0} \rightarrow\left(V_{i 0}, V_{b 0}\right) \equiv\left(0, V_{b 0}\right)$, and applying to the set of Equations (3), (4), and (5), we get

$$
\phi_{1}=\frac{m_{j}}{e}\left\{\left(\frac{\omega}{k}-V_{j 0}\right)^{2}-\frac{\gamma C_{s}^{2} \sigma_{j}}{\left(1+N_{j 1} / N_{j 0}\right)}\right\} \frac{N_{j 1}}{N_{j 0}} .
$$

But $1+N_{j 1} / N_{j 0} \simeq 1$, since $N_{j 1} / N_{j 0} \ll 1$, and therefore 


$$
\varphi_{1}=\frac{m_{j}}{e} \frac{N_{j 1}}{N_{j 0}}\left\{\left(\frac{\omega}{k}-V_{j 0}\right)^{2}-\gamma C_{s}^{2} \sigma_{j}\right\} .
$$

From Poisson's Equation, we have

$$
-\varepsilon_{0}(k)^{2} \varphi_{1}=\frac{e^{2} \varphi_{1}}{k_{B} T_{e}} N_{e 0}(1-\beta)-e N_{j 1}
$$

Finally,

$$
k^{2} \lambda_{\text {Deff }}^{2}+f_{e}(1-\beta)=\sum_{j=i, b} \frac{N_{j 0} / N_{i 0} C_{s}^{2}}{\left(\frac{\omega}{k}-V_{j 0}\right)^{2}-\gamma C_{s}^{2} \sigma_{j}} .
$$

where $f_{e}=\frac{N_{i 0}}{N_{e 0}}$.

Normalizing $k$ with $\lambda_{\text {Deff }}^{-1}, \omega$ with ion plasma frequency $\omega_{p i}$ and $V_{b 0}$ with the ion acoustic speed $C_{s}$ to $u_{b 0}$, Equation (10) can be expressed as

$$
k^{2}+f_{e}(1-\beta)=\frac{1}{\left(\frac{\omega}{k}\right)^{2}-3 \sigma_{i}}+\frac{f_{b}}{\left(\frac{\omega}{k}-u_{b 0}\right)^{2}-3 \sigma_{b}},
$$

where $f_{b}=\frac{N_{0}}{N_{i 0}}$.

\section{Analysis of the Dispersion Behaviour of Linear Waves}

\subsection{Theoretical Analysis}

In the presence of an ion beam, three longitudinal electrostatic waves involving ion motion could propagate; these were, an ion acoustic mode (IA), fast (F) and slow (S) modes. This is in accordance with the experimental observations of [8]. These modes corresponded to $\omega / k>u_{b 0}+\sqrt{3 \sigma_{b}}$ for fast mode, $\omega / k>u_{b 0}-\sqrt{3 \sigma_{b}}$ for slow mode, and $\omega / k>\sqrt{3 \sigma_{i}}$ for ion acoustic mode.

The right hand side (RHS) of Equation (11) is a quartic equation in $\omega$ with the first term on the RHS diverging at $(\omega / k)^{2}=3 \sigma_{i}$, while the second term diverges at $\left(\omega / k-u_{b 0}\right)^{2}=3 \sigma_{b}$. Here the solutions can take, $\omega_{1}=-\sqrt{3 \sigma_{i} k^{2}}, \omega_{2}=\sqrt{3 \sigma_{i} k^{2}}, \omega_{3}=u_{b 0} k+\sqrt{3 \sigma_{b} k^{2}}$, and $\omega_{4}=u_{b 0} k-\sqrt{3 \sigma_{b} k^{2}} . \omega_{1}, \omega_{2}, \omega_{3}$ and $\omega_{4}$ are the assymptotes in Figure 2. From the same figure it was observed that when, $(\omega / k)^{2} \rightarrow 3 \sigma_{i}$ or $\left(\omega / k-u_{b 0}\right)^{2} \rightarrow 3 \sigma_{b}$, the RHS $\rightarrow \infty$ in Equation (11), gives rise to the two stream instabilities between ion and ion beam. Between $\omega_{2}$ and $\omega_{3}$, there exists a minimum value $\left(F\left(\omega_{m}\right) \simeq 2.6758\right)$, that corresponds to $\mathrm{d} F(\omega) / \mathrm{d} \omega=0$, above which the wave is unstable. The maximum values exist between $\omega_{1}-\omega_{2}$ and $\omega_{3}-\omega_{4}$ at $F\left(\omega_{\max }\right) \simeq-10.246$ and $F\left(\omega_{\max }\right) \simeq-1.671$, respectively. The wave exists in the range $-1.671<F(\omega)<2.6758$.

Since the coefficients of Equation (11) are real, there are two complex roots which are complex conjugates to each other, i.e., $\omega_{r} \pm i \omega_{i}$ where $\omega_{r}$ is the real part of frequency and $\omega_{i}$ is assumed to be positive and is known as the growth rate [9]. The complex conjugate roots were obtained for $\omega<\omega_{1}$ and $\omega>\omega_{4}$ as $\simeq(-0.197308 \pm 0.221603 i)$. The complex root with positive imaginary part gives rise to two stream instability with a growth rate of $\omega_{i}=0.221603$.

Further theoretical analysis of the dispersion relation (Equation (11), revealed that, in the limiting case of $f_{b}=0$, DIA solitary waves propagated in the beam with a phase velocity $V_{p}=\omega / k$, given by 


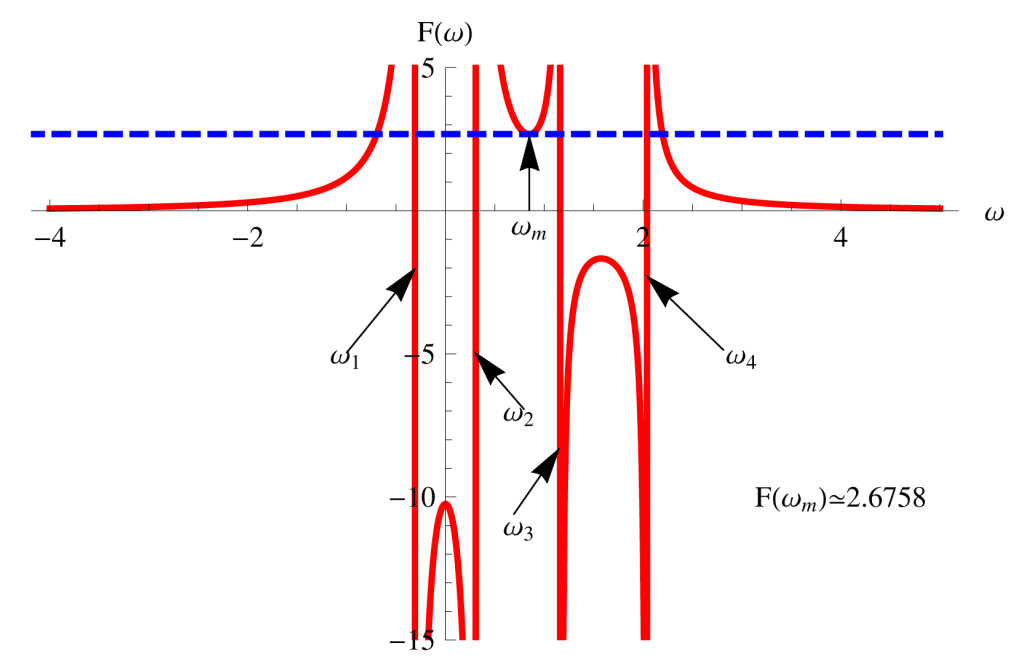

Figure 2. Plot showing dispersion relation.

$$
V_{p}=\sqrt{3 \sigma_{i}+\frac{1}{k^{2}+f_{e}(1-\beta)}} .
$$

This implies that the phase speed of an ion acoustic wave increased in the prescence of nonthermal electrons. However, in the abscence of nonthermal electrons, i.e., $\beta=0$, it yields

$$
V_{p}=\sqrt{3 \sigma_{i}+\frac{1}{k^{2}+f_{e}}},
$$

which in the long wavelength limit, i.e., $k \ll 1$, gives

$$
V_{p}=\sqrt{3 \sigma_{i}+1 / f_{e}} \text {. }
$$

For cold ions, $\sigma_{i}=0$, we obtain

$$
V_{p}=\sqrt{1 / f_{e}} .
$$

This is similar to the phase speed obtained by [10], for an electron-ion plasma with cold dust.

\subsection{Numerical Analysis}

Numerical examination of the dispersion relation in Equation (11) for the three wave modes propagating along the beam is shown in Figure 3. The effect of ion beam density ratio ( $\mathrm{fb}$ ), ion beam speed (ub0), ion beam (sgb) and ion (sgi) temperature ratio, on the frequency of the IA, F and S modes are presented. It was found that, as the ion beam speed increased, (Figure 3(a)) the phase speed of both the F- and S-modes increased while the IAmode remained unchanged. The same observation was made for F- and IA-mode when ion beam temperature ratio was increased (Figure 3(b)). This is in accordance with the findings of [3]. For the S-mode, increasing ion beam temperature ratio decreased the phase speed. However, in contrast to (Figure 3(a)), the effect was greater with increasing ion beam speed. This has a physical sence, since ion beam speed is the source of energy that drives the instability. Therefore, ion beam speed has an effect of enhancing the phase speeds of $\mathrm{F}$ and $\mathrm{S}$ modes. Morestill, it was also found out that, ion beam density ratio had no effect on the phase speed of all modes (Figure 3(c)). Finally, increasing the ion beam temperature ratio only affected the IA-mode as shown in Figure $3(d))$.

\section{Conclusions}

The study findings show that on close examination of the derived dispersion relation there are three longitudinal electrostatic modes involving ion motion that propagates. These were ion acoustic, fast and slow modes. 


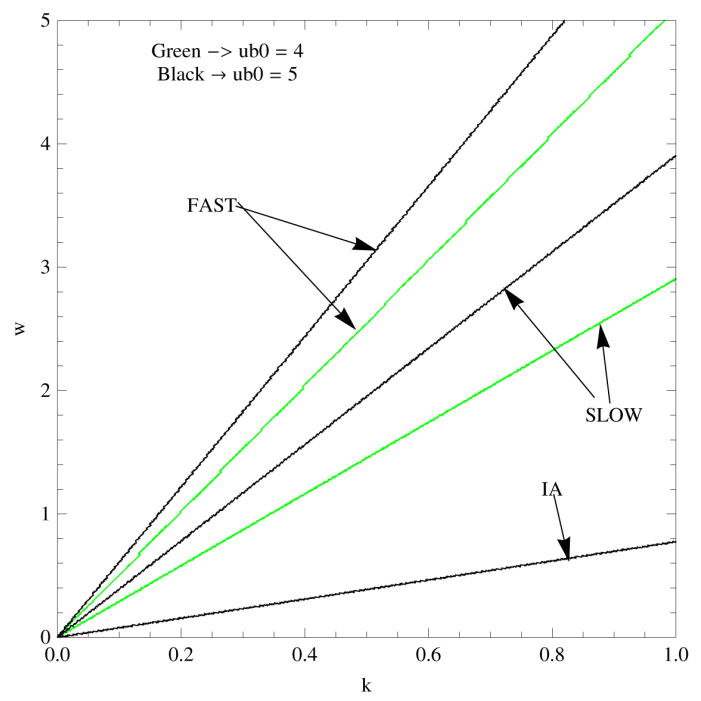

(a)

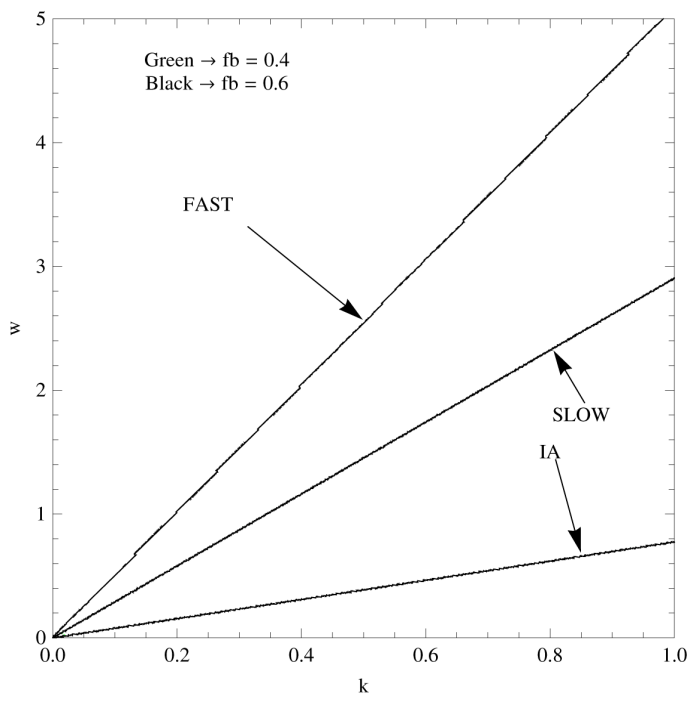

(c)

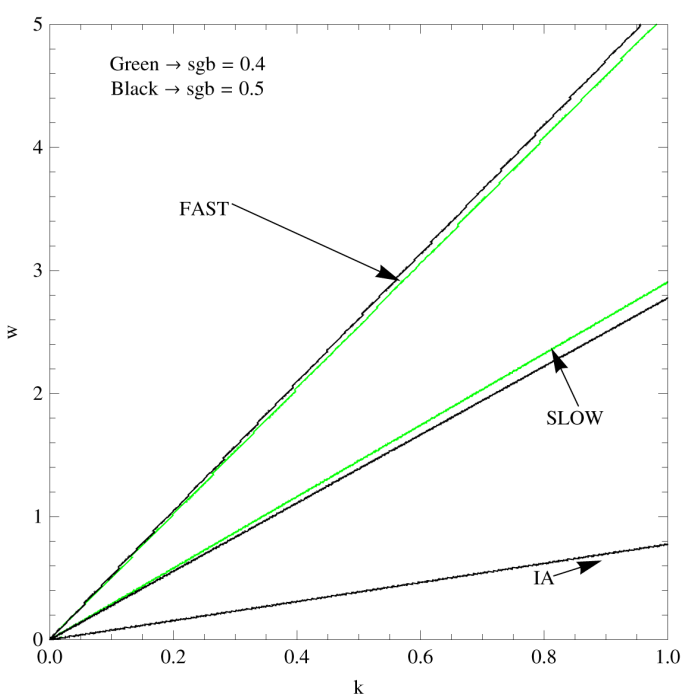

(b)

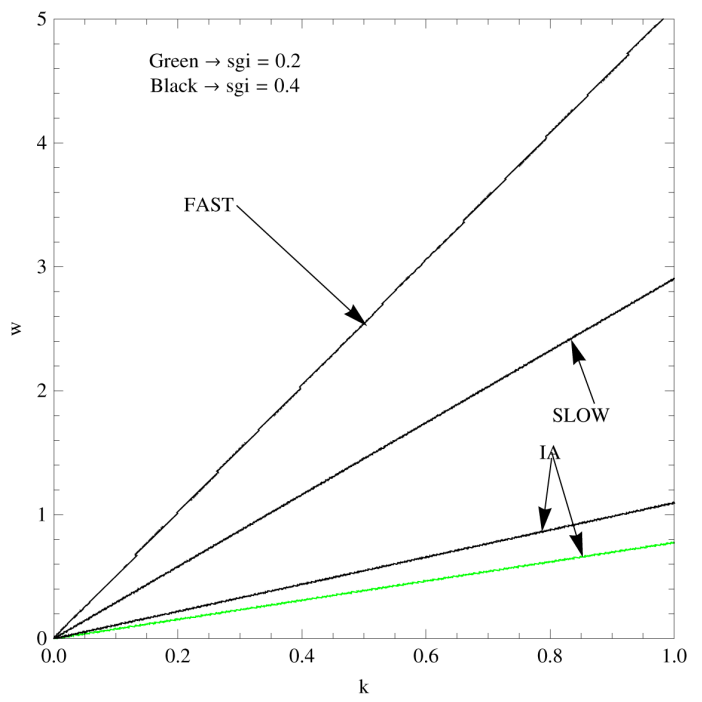

(d)

Figure 3. Contour plot showing dispersion relation against the wave number $k$ for Fast (F), Slow (S), and Ion Acoustic (IA) modes for different parameter values. Top Panel: (a) $\mathrm{fb}=0.4$, sgi $=0.2, \mathrm{sgb}=0.4$ with values of ub0 $=4,5$; (b) sgi $=0.2$, $\mathrm{sgb}=0.4$, ub0 $=4$ with values of $\mathrm{sgb}=0.4,0.5$. Bottom Panel: (c) $\mathrm{fb}=0.4, \mathrm{sgb}=0.4, \mathrm{ub} 0=4$ with values of $\mathrm{fb}=0.4,0.6$ and (d) $\mathrm{fb}=0.4$, sgi $=0.2, \mathrm{ub} 0=4$ with values of sgi $=0.2,0.4$.

Thus for the two stream instability to occur between ion and ion beam, then it's $(\omega / k) \rightarrow \sqrt{3 \sigma_{i}}$ or when $\left(\omega / k-u_{b 0}\right) \rightarrow \sqrt{3 \sigma_{b}}$. Increasing the ion beam speed increased the phase speed of both the F- and S-modes, while the IA-acoustic mode remained unaffected. Ion beam temperature changes have the same effect but slightly less as compared to ion beam speed. Ion beam density ratio has no effect on the phase speed of all modes while ion beam temperature ratio affected the IA- mode only.

These theoretical findings could be useful in determining onset of instability in laboratory ion beam driven plasmas as well as space plasmas.

\section{Acknowledgements}

Author 1 acknowledges the funding from East African Astronomical Research Network (EAARN) supported by International Science Program (ISP). 


\section{References}

[1] Okutsu, E., Nakamura, M., Nakamura, Y. and Itoh, T. (1978) Amplification of Ion-Acoustic Solitons by an Ion Beam. Plasma Physics, 20, 561. http://dx.doi.org/10.1088/0032-1028/20/6/006

[2] Adhikary, N.C., Misra, A.P., Bailung, H. and Chutia, J. (2010) Ion-Beam Driven Dust Ion-Acoustic Solitary Waves in Dusty Plasmas. Physics of Plasmas, 17, Article ID: 044502. http://dx.doi.org/10.1063/1.3381036

[3] Misra, A.P. and Adhikary, N.C. (2011) Large Amplitude Solitary Waves in Ion-Beam Plasmas with Charged Dust Impurities. Physics of Plasmas, 18, Article ID: 122112. http://dx.doi.org/10.1063/1.3671951

[4] Cairns, R.A., Mamun, A.A., Bingham, R., Dendy, R.O., Nairn, C.M.C. and Shukla, P.K. (1995) Electrostatic Solitary Structures in Non-Thermal Plasmas. Geophysical Research Letters, 22, 2709-2712. http://dx.doi.org/10.1029/95GL02781

[5] Baluku, T.K. and Hellberg, M.A. (2011) Ion Acoustic Solitary Waves in an Electron-Positron-Ion Plasma with Non-Thermal Electrons. Plasma Physics and Controlled Fusion, 53, Article ID: 095007. http://dx.doi.org/10.1088/0741-3335/53/9/095007

[6] Verheest, F. and Pillay, S.R. (2008) Large Amplitude Dust-Acoustic Solitary Waves and Double Layers in Nonthermal Plasmas. Physics of Plasmas, 15, Article ID: 013703. http://dx.doi.org/10.1063/1.2831025

[7] Sabry, R., Moslem, W.M. and Shukla, P.K. (2009) Fully Nonlinear Ion-Acoustic Solitary Waves in a Plasma with Positive-Negative Ions and Nonthermal Electrons. Physics of Plasmas, 16, Article ID: 032302. http://dx.doi.org/10.1063/1.3088005

[8] Gresillon, D. and Doveil, F. (1975) Normal Modes in the Ion-Beam-Plasma System. Physical Review Letters, 34, 7780. http://dx.doi.org/10.1103/PhysRevLett.34.77

[9] Gurnett, D.A. and Bhattacharjee, A. (2005) Introduction to Plasma Physics with Space and Laboratory Applications. Cambridge University Press, Cambridge, 1-3, 87-93. http://dx.doi.org/10.1017/CBO9780511809125.002

[10] Shukla, P.K. and Silin, V.P. (1992) Dust Ion-Acoustic Waves. Physica Scripta, 45, 508.

http://dx.doi.org/10.1088/0031-8949/45/5/015 